\title{
Theoretical Equivalence in Classical Mechanics and its relationship to Duality
}

\author{
Nicholas J. Teh \\ Department of Philosophy \\ Notre Dame University \\ nteh@nd.edu
}

\author{
Dimitris Tsementzis \\ Department of Philosophy \\ Princeton University \\ dtsement@princeton.edu
}

September 28, 2015

\begin{abstract}
As a prolegomenon to understanding the sense in which dualities are theoretical equivalences, we investigate the intuitive 'equivalence' of hyper-regular Lagrangian and Hamiltonian classical mechanics. We show that the symplectification of these theories (via Tulczyjew's Triple) provides a sense in which they are (1) isomorphic, and (2) mutually and canonically definable through an analog of 'common definitional extension'.
\end{abstract}

\section{Contents}

1 Introduction 1

1.1 Duality as Theoretical Equivalence $\ldots \ldots \ldots \ldots \ldots \ldots$. . . . . . . . . . 2

1.2 Theoretical Equivalence in the Philosophy of Science . . . . . . . . . . . . . . . . 3

1.3 Strategy and Thesis $\ldots \ldots \ldots \ldots \ldots \ldots$

\begin{tabular}{|ll|l}
2 & Tulczyjew's symplectification of classical mechanics & 5
\end{tabular}

2.1 Review of Classical Mechanics . . . . . . . . . . . . . . . . . . . 5

2.2 Tulczyjew's reformulation $\ldots \ldots \ldots \ldots \ldots \ldots \ldots$

\begin{tabular}{lll}
\hline 3 & Equivalence regained & 11
\end{tabular}

3.1 Common Definitional Extensions . . . . . . . . . . . . . . . . . . . . . . . . . 11

3.2 A First Analogy . . . . . . . . . . . . . . . . . . . . . . . . . . . . . . . . 14

3.3 A Second Analogy . . . . . . . . . . . . . . . . . . . . . . . . . . . 15

3.4 Naturality of the Tulczyjew Triple $\ldots \ldots \ldots \ldots$. . . . . . . . . . . . 17

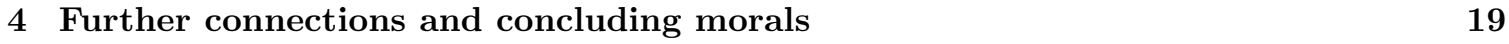

\section{Introduction}

In this paper, we will show that a careful consideration of the relationship between 'physical dualities' and the more general philosophical theme of 'theoretical equivalence' leads to a deeper understanding 
of both topics. We do so by focusing on the equivalence between the hyper-regular case of classical Lagrangian and Hamiltonian mechanics: call this CM-Equiv. On the one hand, this example offers us insight into what it might mean for two dual theories to be theoretically equivalent, in a sense that goes beyond the (admittedly impressive) 'dictionary correspondences' which are characteristic of a physical duality. On the other hand, our work will bring the (in many cases highly abstract) philosophical discussion about theoretical equivalence to bear on a concrete physical example, viz. CM-Equiv.

The rest of this introduction focuses on the theme of 'theoretical equivalence'. Section 1.1 recalls the fact that exact dualities are theoretical equivalences, and Section 1.2 reviews the discussion of theoretical equivalence in the philosophy of science literature. Section 1.3 then lays out our strategy for showing that the correspondence between (hyper-regular) Hamiltonian and Lagrangian mechanics is a theoretical equivalence.

In Section 2, we briefly review the mathematical background to our discussion. Section 2.1 recalls the 'standard' framework of classical mechanics and considers problems with describing theoretical equivalence within this formalism. Section 2.2 then sketches Tulczyjew's (cf. Tulczyjew, 1977) and related work) reformulation of classical mechanics, which we take to be an improvement on the standard framework.

In Section 3, we argue that Tulczyjew's reformulation can be interpreted as establishing a theoretical equivalence between Hamiltonian and Lagrangian mechanics (CM-Equiv). Section 3.1 reviews the notion of Common Definitional Extension (CDE) which is often deployed in the philosophy of science literature as a criterion of theoretical equivalence. In Sections 3.2 and 3.3, we go on to discuss two ways in which Tulczyjew's results can be interpreted as saying that Lagrangian and Hamiltonian mechanics have a CDE (these correspond to the two different notions of 'theory' - T1 and T2 discussed in Section 2.1 below). Section 3.4 then discusses the relationship between (CM-Equiv) and various themes from category theory. In particular, we discuss how the Tulczyjew triple is natural in a category-theoretic sense and explain how this points to an analogy with the notion of Morita equivalence in ring theory.

Finally, Section 4 lists the rich connections and analogies between our interpretation of CM-Equiv and more sophisticated notions of duality in physics.

\subsection{Duality as Theoretical Equivalence}

As discussed by the other articles in this issue, modern physics - especially string theory and QFT - has uncovered a spectacular array of dualities ${ }^{1}$ These take the form of an (in many cases only conjectured) 'equivalence' between the dual theories, which manifests itself as a bijection (also called a 'dictionary correspondence' or 'duality map') between the physical quantities (and states) of each theory.

This bijection between physical quantites has justly attracted philosophical attention because of its remarkable features. For instance, it often exchanges the weak and strong coupling regimes of a pair of seemingly different theories, thus raising interpretive questions about how we should understand 'fundamentality' in such theories. On the other hand, it is clear that the bijection between physical quantities does not by itself constitute a theoretical equivalence, since it does not tell us how to map the entire (mathematical) structure of one theory to another. One can thus pose a fundamental philosophical question that goes beyond the dictionary aspect of dualities:

\footnotetext{
${ }^{1}$ See Polchinski, 2015] in this volume for an overview.
} 
(Dual-Equiv) What does it mean for a pair of dual theories to be equivalent?

In what follows, we will approach Dual-Equiv by focusing on a special case, viz. the equivalence between (hyper-regular) Lagrangian and Hamiltonian mechanics, which we have called CM-Equiv above. Of course, CM-Equiv is not usually characterized as an instance of physical duality, due to its being too elementary. Nonetheless, the problem of adequately characterizing the equivalence between these simple theories is less simple than what one might imagine, and its resolution brings together interesting tools from symplectic geometry and category theory. It may also provide a template for how to approach theoretical equivalence in more sophisticated cases.

Furthermore, our analysis of CM-Equiv illustrates how one might begin to connect the topic of duality to more general discussions about 'theoretical equivalence' in the philosophy of science, to which we turn in Section 1.2.

\subsection{Theoretical Equivalence in the Philosophy of Science}

The question of theoretical equivalence has two parts: (A) What is a scientific theory?; which in turn allows us to pose: (B) When are two scientific theories equivalent? A long and often tortuous literature has grown out of all the attempts to give satisfactory answers to (A) and (B).

Beginning with the logical positivists, through Carnap and Hempel, the so-called syntactic view of theories was developed ${ }^{2}$ Roughly, this view answers (A) by claiming that a theory is a collection of theorems expressed in a fixed formal language, and it answers (B) by claiming that two theories are equivalent iff they are logically equivalent, in the sense that each theory's axioms can be derived from the other's. There were several problems with this view. First, undue emphasis was placed on attempting to spell out the scientific application of properties of formal systems (e.g. axiomatizability, decidability, interpolation etc.). Secondly, and more importantly for our purposes, identifying two scientific theories only if they are logically equivalent proves far too restrictive: in particular, this means that one cannot even compare two theories that are formulated using different vocabularies (i.e. have different signatures in the model-theoretic sense.)

In reaction to the problems with the syntactic view, the semantic view of theories was developed by, among others, van Fraassen, Suppe and Glymour (cf. e.g. van Frassen, 1980, Suppe, 1989). Roughly speaking, this view holds, in answer to (A), that a theory is a class of models, and the theory is true iff one of these models is 'isomorphic' to the actual world. Thus, in answer to (B), the semantic view holds that two such theories will be considered equivalent iff they have identical classes of models. There are also several problems with this view but the one that concerns us the most is the following: What is a good notion of 'equivalence' between two classes of models? In other words, what kind of relation must these classes stand in if we are to consider them equivalent:4

This all-too-brief sketch ${ }^{5}$ is sufficient to show that efforts to answer the question of theoretical equivalence have resulted in a dialectical limbo. For both the last century's major approaches to answering this question seem to face insurmountable difficulties. But our aim here is neither to find some dialectical middle ground nor to ameliorate any of the currently avaiable options. Instead, we are motivated by a more practical challenge: the philosophy of science has still not been able

\footnotetext{
${ }^{2}$ Also once called the 'received view', cf. e.g. Suppe, 1989].

${ }^{3}$ In particular, what does it mean for a model to be isomorphic to the world? What kind of epistemic import do non-world-isomorphic models have? And so on.

${ }^{4}$ For a thorough examination of these issues as well as a proposed strategy to tackle them cf. Halvorson, 2012 .

${ }^{5}$ For a more thorough overviw of the debate, cf. Suppe, 1998
} 
to provide a fruitful and appealing framework for conceptualizing the simplest cases of intuitive theoretical equivalence' in physics. So if a link is to be drawn between the question of theoretical equivalence in the philosophy of science, on the one hand, and ongoing research on dualities in physics, on the other, then one should first develop the resources to address simple cases such as classical mechanics.

\subsection{Strategy and Thesis}

In order to address this practical challenge, this paper will describe a concrete and satisfactory framework for CM-Equiv. This will serve as a prolegomenon to developing a general framework for theoretical equivalence in the philosophy of science, and thus to the study of more sophisticated dualities.

By 'satisfactory', we have in mind two desiderata:

- S1: Our framework should address the question of how to construct an appropriate mathematical notion of equivalence (e.g. 'isomorphism' or any of its ( $n$-)categorical generalizations) between dual (or 'intuitively equivalent') physical theories.

- S2: Our framework should satisfy certain independently-motivated criteria from the philosophy of science about what it means for two theories to be 'equivalent'.

Even within the elementary setting of classical mechanics, the task of exhibiting a framework which satisfies S1 and S2 is decidedly non-trivial. Let us briefly sketch how these desiderata arise in our work.

First, S1. One might imagine classical mechanics to be perfectly well-understood. But philosophers have managed to make trouble even in the mechanical paradise of our 19th century precursors: witness the recent debate between North, 2009 and Curiel, 2014 over whether Lagrangian or Hamiltonian mechanics has conceptual (and/or metaphysical) priority. North argues in favor of the priority of the Hamiltonian theory, whereas Curiel defends the priority of the Lagrangian theory. Our interest is not in adjudicating this debate, but instead in highlighting a common (and by our lights mistaken in the hyper-regular case) assumption shared by both sides, viz.

Hamiltonian mechanics is not isomorphic to Lagrangian mechanics ${ }^{6}$

It is somewhat puzzling that this premise should be found so convincing. For not only has there been no demonstration that there cannot be an 'isomorphism', but in addition, it is entirely unclear how one would formulate an isomorphism between the two to begin with. After all, it is a mathematical truism that the detailed content of the concept 'isomorphism' depends on the particular category $\sqrt{7}$ that one is in, and attempts to construct such a category for classical mechanics have been wanting 8 Thus, any attempt to resolve the question of whether Hamiltonian mechanics

${ }^{6}$ Curiel, 2014] says explicitly that the geometric structures of Hamiltonian and Lagrangian mechanics are not 'isomorphic', and North, 2009 expresses the same idea when she says that they are 'different mathematical structures'. Note, however, that while it seems that these authors would hold this of the hyper-regular case, their general discussion is conducted without assuming hyper-regularity; see e.g. footnote nr. 50 of [North, 2009] (we are grateful to an anonymous referee for pointing this out).

${ }^{7} \mathrm{Or}$, if one wants to avoid using the language of categories here, we can say instead "depends on the particular structure one cares to study in the objects of interest."

${ }^{8}$ More precisely: in an arbitrary category, an isomorphism between objects $A$ and $B$ is defined by the existence of morphisms $i: A \rightarrow B, j: B \rightarrow A$ such that $j i=1_{A}$ and $i j=1_{B}$, but the detailed criteria of 'sameness' depends on the further structure of such morphisms (i.e. whether they are diffeomorphisms, homomorphisms, etc). 
can be isomorphic to Lagrangian mechanics must either (i) exhibit an appropriate category in which 'isomorphism' is understood, or (ii) show that no such category exists. In what follows, we argue that Tulczyjew's framework for classical mechanics provides an adequate notion of 'isomorphism' between the hyper-regular case of these theories. In particular, the isomorphism is an equality between two relevant structures, one emerging from the Lagrangian side and one from the Hamiltonian side.

Next, S2. How do we know that our proposed 'framework for isomorphism' counts as a case of theoretical equivalence, in the sense that has been widely discussed in the philosophy of science literature? In order to establish this, we will show that if one takes seriously a standard logical construal of the notion of theoretical equivalence, one is led to a very strong category-theoretic analogy with the structure that we uncover in Tulczyjew's approach to classical mechanics. Furthermore, this analogy suggests that an appropriate framework for theoretical equivalence will combine aspects of both the syntactic and the semantic view of theories.

To sum up, our central thesis is that Tulczyjew's formulation of classical mechanics, as laid out in Section 2.2 and re-interpreted in Section 3, satisfies both S1 and S2.

\section{Tulczyjew's symplectification of classical mechanics}

In this section we describe Tulczyjew's work on the Legendre transformation, which is based on his insight that the very concept of a classical mechanical system (and not merely the phase space of that system) should be described in terms of symplectic geometry.

In Section 2.1 we briefly review some standard concepts from classical mechanics and emphasize that, although one can try to use these materials to construct an isomorphism between the Hamiltonian and Lagrangian theory, such attempts are flawed. In Section 2.2, we sketch Tulczyjew's theory and show that it provides a better notion of 'equivalence' between Lagrangian and Hamiltonian mechanics.

\subsection{Review of Classical Mechanics}

For our purposes, it will suffice to consider only the simplest scenario in classical mechanics. Let $Q$ be physical configuration space manifold, in which the particles live. There are then two alternative representations of classical mechanics:

- Hamiltonian mechanics, which is specified by the cotangent bundle $T^{*} Q$ and some $H \in$ $C^{\infty}\left(T^{*} Q\right)$

- Lagrangian mechanics, which is specified by the tangent bundle $T Q$ and some $L \in C^{\infty}(T Q)$.

At this point it will be helpful to discuss the use of the terms 'theory' and 'model' in classical mechanics. Of course, there is no unified and consistent use of 'theory' by practitioners of physics the meaning of the term varies depending on the domain of physics that one is considering, and the application that one has in mind. Consequently, there is usually a very vague understanding of the distinction between 'model' and 'theory' and exactly what it means for something to be a model of a (physical) theory. In the case of (classical) Lagrangians and Hamiltonians, it is common to say that: 
(T1) A theory is specified by a particular Lagrangian/Hamiltonian, and a model of this theory is a solution to the equations of motion derived from that particular Lagrangian/Hamiltonian?

This will correspond to our analogy with the syntactic view of theories in Section 3.2.

It will also be useful to have in mind a more general use of the term 'theory', which refers to all the different forms of (Lagrangian/Hamiltonian) classical mechanics that one can formulate with respect to some configuration space $Q$ :

(T2)A theory is identified with the set of possible Lagrangians/Hamiltonians on $T Q$ and $T^{*} Q$ respectively. A model of such a theory is some particular Lagrangian/Hamiltonian out of this set.

This will correspond to our analogy with the semantic view of theories in Section 3.3.

As emphasized in the introduction, this paper will be exclusively concerned with the hyperregular case of classical mechanics, where the 'bundle Legendre transformation' $\mathcal{L}_{L}$ is a global diffeomorphism ${ }^{10} \mathcal{L}_{L}$ is defined as follows, given the choice of some particular Lagrangian $L$ :

$$
\mathcal{L}_{L}(x, \cdot): T_{x} Q \rightarrow T_{x}^{*} Q, \quad\left\langle\mathcal{L}_{L}(x, v), w\right\rangle:=d\left(\left.L\right|_{T_{x} Q}(v)\right)(w)=\left.\frac{d}{d t}\right|_{t=0} L(x, v+t w)
$$

It is a familiar fact that there is no canonical isomorphism between a vector bundle and its dual, in the sense that one has to make a further choice (beyond specifying the dual objects) in order to obtain an isomorphism. $\mathcal{L}_{L}$ involves such a choice (viz. a choice of $L$ ) in the above pairing $\langle\cdot, \cdot\rangle$ between vectors, on the one hand, and covectors that take the form of $d L$ restricted to a point $v \in T_{x} Q$, on the other. This thus leads to an $L$-dependent fiber bundle isomorphism between $T Q$ and $T^{*} Q$.

$\mathcal{L}_{L}$ in turn leads to the more familiar functional sense of the Legendre transformation (and its inverse), i.e.

$$
H(\mathcal{L}(x, v))=\left\langle\mathcal{L}_{L}(x, v), v\right\rangle-L(x, v) .
$$

(1), (2) are used, in the hyper-regular case of Lagrangian/Hamiltonian mechanics, to colloquially express our idea of T1-'equivalence'. Indeed, this equivalence is typically taken to consist in the bijection between Hamiltonians and Lagrangians provided by (2), given a fixed configuration space $Q$. Furthermore, one sees that the states $(q, \dot{q}) \in T Q$ and $(q, p) \in T^{*} Q$ are paired by writing out the local form of (1). One is thus in an analogous situation to that of most dualities, where one has a 'dictionary' bijection between the physically relevant states and quantities.

Let us now turn to the question of what one would need in order to use these standard materials to show that Lagrangian mechanics is 'equivalent' to Hamiltonian mechanics. We will explore two strategies to demonstrate this. The first is an example of treating a theory in the manner of T1, and the second is an example of treating a theory in the manner of $\mathrm{T} 2$.

The first strategy is to take isomorphism as our standard of T1-equivalence. In other words, we should fix $Q$ and find a category that contains both $(T Q, L)$ and its Legendre transform $\left(T^{*} Q, H\right)$ as objects, such that the Legendre transform provides an isomorphism between them. Unfortunately,

\footnotetext{
${ }^{9}$ Implicit in the physicist's identification of a theory $\mathbb{T}$ with $L / H$ is the understanding that this data is sufficient to construct a theory $\mathbb{T}^{\prime}$, given by the equations of motion associated with $L / H$. Solutions are thus associated with $\mathbb{T}$ via $\mathbb{T}^{\prime}$.

${ }^{10}$ In a more familiar language to physicists: where the Hessian of the relevant Lagrangian is non-singular, i.e. its determinant is non-zero.
} 
this tack immediately runs afoul of the problem that $(T Q, L)$ and $\left(T^{*} Q, H\right)$ are very different structures, and thus cannot be housed together in a category that has a natural and informative notion of morphism. A facetious way of dealing with the issue would of course be to define a category with only two objects, which represent $(T Q, L)$ and $\left(T^{*} Q, H\right)$ respectively; we then say that there is a morphism between the objects just in case there is a Legendre transformation between $(T Q, L)$ and $\left(T^{*} Q, H\right)$, and proclaim that composing these morphisms gives the identity morphism on the relevant object. But this is no solution: it only serves to highlight the importance of choosing an 'appropriate' category in order to render a proposed standard of equivalence interesting or informative.

The second strategy is to advert to a categorical generalization of isomorphism in order to formalize T2-equivalence. For instance, one might construct a categorical equivalence between a Hamiltonian category $\operatorname{Ham}(\mathbf{Q})$ and a Lagrangian category $\mathbf{L a g}(\mathbf{Q})$ (whose objects are to be thought of as 'models' of Hamiltonian and Lagrangian mechanics respectively). $\operatorname{Ham}(\mathbf{Q})$ and $\mathbf{L a g}(\mathbf{Q})$ would be the categories of models of the (T2)-theories of Hamiltonian and Lagrangian mechanics, and one might propose that the objects of $\operatorname{Ham}(\mathbf{Q})$ (with $Q$ fixed) are all the pairs $\left(T^{*} Q, H\right)$ for any $H$ on $T^{*} Q$, and that only trivial morphisms exist; mutatis mutandis with the definition of $\operatorname{Lag}(Q)$. One then defines a pair of 'Legendre transform' functors $F$ and $G$ such that $F(T Q, L)=\left(T^{*} Q, H\right)$ by means of 1,2 (mutatis mutandis for its quasi-inverse functor $G$ ), thus providing a notion of T2-equivalence between Hamiltonian and Lagrangian theories.

Unfortunately, this strategy is not satisfactory, for two reasons. First, the eq. (1) part of the Legendre transform functor is not canonical, in the sense that the diffeomorphism between $T Q$ and $T^{*} Q$ depends on a further choice, viz. $L$. Second, while the map $(2)$ induces a bijection between the set of Lagrangians on $T Q$ and the set of Hamiltonians on $T^{*} Q$, this map does not itself seem to be 'structure-preserving' in any clear way. One would ideally like (2) to be characterized in such a way as to make transparent what the important structural feature of Hamiltonians/Lagrangians is, and how the preservation of this structure induces a bijection between Lagrangians and Hamiltonians for a fixed $Q 11$

We will soon see that Tulcyjew's work remedies both defects: First, he constructs a canonical correspondence between T2-theories; and second, each Lagrangian/Hamiltonian is represented as a geometric object (i.e. a Lagrangian submanifold), whose geometry is preserved by a symplectomorphism between the objects representing Lagrangians, and the objects representing Hamiltonians.

\subsection{Tulczyjew's reformulation}

In the 1970s (cf. Tulczyjew, 1974 and [Tulczyjew, 1977]), Wlodzimierz M. Tulczyjew found a way of reformulating classical mechanics that gave a geometrical interpretation not just to the space of solutions of a theory, but indeed to the notion of a theory itself.

In order to appreciate his achievement, consider that a space equipped with some algebraic structure typically contains a separation between two aspects/levels in its description: the space is thought of as carrying the algebraic structure (e.g. a metric or a form) at every point, and a structurepreserving map of the space is conceived as an isomorphism of the space (e.g. a diffeomorphism if we are in the smooth category) that preserves the algebraic structure at every point.

An analog of this separation exists in the case of mechanics, i.e. between: on the one hand, the space in which curves describe the evolution of a system, and on the other hand, the equations of

\footnotetext{
${ }^{11}$ It is instructive to compare the second strategy with something like Gelfand duality, which is also an equivalence of categories. The chief difference is that the latter provides a complete reconstruction of objects in one category from objects in the other.
} 
motion (i.e. the structural conditions) which must be satisfied by such curves. Tulczyjew managed to overcome this separation by describing a (Hamiltonian or Lagrangian) mechanical system as a submanifold in an abstract space even larger than the original evolutionary space, in such a way that curves on this space satisfy the relevant equations of motion in virtue of the space's geometry together with a pair of canonical isomorphisms, referred to as $\alpha$ and $\beta$ below.

Interestingly, the set of tools needed to implement Tulczyjew's approach was of a piece with a much grander movement within geometry, viz. the idea that there should be symplectic analogs of objects in other geometries, and that such correspondences could be exploited to study geometry more generally. In order to achieve this program of 'symplectification', symplectic versions of all sorts of other objects were found. This procedure ranges from the relatively straightforward (e.g., making a manifold 'symplectic' by applying the cotangent functor into it, which is the first step of Tulczyjew's approach) to constructions such as the symplectification of a contact manifold. A related idea - articulated subsequently to Tulczyjew's work, but providing an ideological context for it - was Weinstein's 'Lagrangian creed' Weinstein, 1981, viz. the idea that all important concepts in symplectic geometry should be expressed in terms of Lagrangian submanifolds. For, as we shall see shortly, in Tulczyjew's approach, a mechanical system turns out to be a Lagrangian submanifold of an appropriately defined space.

Let us now turn to the mathematical details of Tulczyjew's approach. The key insight comes from the theory of double vector bundles, viz.: although there is no canonical isomorphism between a real vector bundle $M($ e.g. $T Q)$ and its dual $M^{*}\left(\right.$ e.g. $\left.T^{*} Q\right)$, there is a canonical isomorphism - indeed a symplectomorphism - between $T^{*} M$ and $T^{*} M^{*}$. In what follows, we shall use $Q$ to denote the physical configuration space of a mechanical system, so the real vector bundles $M \equiv T Q$ and $M^{*} \equiv T^{*} Q$ will denote the usual Lagrangian and Hamiltonian state spaces respectively. The lack of canonicity will then be overcome by lifting the notion of a T1-theory into $T^{*} M$ and $T^{*} M^{*}$ respectively.

The canonical symplectomorphism $\kappa_{M}: T^{*} M^{*} \cong T^{*} M$ can be constructed using purely categorytheoretic arguments, for which we refer the reader to [Meng, 2015 for details. For our purposes, it will suffice to record the local form of this symplectomorphism, viz.

$$
\kappa_{M}:(q, \alpha, p, \hat{u}) \mapsto(q,-\hat{u}, p, \alpha),
$$

where $(q, \alpha)$ are coordinates on $M^{*}$, and $(p, \hat{u})$ are coordinates on a fiber of $T^{*} M^{*} 12$ One can directly check that this is a symplectomorphism by showing that, under this transformation, the Liouville 1-forms on $T^{*} M^{*}$ and $T^{*} M$ (which are mapped to each other) respectively define the same symplectic structure.

Before going on to discuss how Tulczyjew proposes to 'lift' mechanics to the level of $T^{*} M$ and $T^{*} M^{*}$, let us recall some standard notions from symplectic geometry. Let $(P, \omega)$ be a symplectic manifold. An isotropic submanifold $N$ of $P$ is a submanifold of $P$ such that the pullback of $\omega$ along the inclusion $N \hookrightarrow P$ is zero. A Lagrangian submanifold of $P$ is an isotropic submanifold of half the dimension of $P$ (i.e. a maximal isotropic submanifold).

The next notion was introduced by Tulczyjew in order to describe how an abstract symplectic manifold can have different, and yet symplectomorphic descriptions, viz. as cotangent bundles of some other manifold. Note that we use the notation $\pi_{M}: T^{*} M \rightarrow M$ for the natural cotangent bundle projection.

\footnotetext{
${ }^{12}$ The non-standard notation follows [Meng, 2015] and has been chosen for ease of comparison.
} 
Definition 2.2.1. A special symplectic manifold is a quadruple $S=(P, M, \pi, \theta)$ where $\pi: P \rightarrow$ $M$ is a smooth fiber bundle and $\theta$ a one-form on $P$, subject to the following condition: there exists a diffeomorphism $\alpha: P \rightarrow T^{*} M$ such that $\pi=\pi_{M} \circ \alpha$ and $\theta=\alpha^{*} \theta_{M}$, where $\theta_{M}$ is the Liouville form on $T^{*} M$.

It follows from the definition of a special symplectic manifold $S$ that $(P, d \theta)$ is a symplectic manifold that is symplectomorphic (via $\alpha)$ to $\left(T^{*} M, \omega_{M} \equiv d \theta_{M}\right)$. It is thus natural to call $(P, d \theta)$ the underlying symplectic manifold of $S$.

The motivation behind this definition stems from the following relationship between an underlying symplectic manifold and two different cotangent bundles:

Definition 2.2.2. The Tulczyjew triple consists of the following (anti-)symplectomorphisms, for any manifold $Q$

$$
T^{*} T Q \stackrel{\alpha_{Q}}{\leftarrow} T T^{*} Q \stackrel{\beta_{Q}}{\rightarrow} T^{*} T^{*} Q
$$

Here, $\beta_{Q}$ is defined as the canonical isomorphism between $T T^{*} Q$ and $T^{*} T^{*} Q$ that one obtains from the (non-degeneracy of the) symplectic form $\omega_{Q}$ on $T^{*} Q$. $\alpha_{Q}$, on the other hand, is defined as $\kappa_{T Q} \circ \beta_{Q}$, where $\kappa_{T Q}$ is the canonical symplectomorphism of (3) for $M \equiv T Q$.

Although it might not be immediately obvious that $T T^{*} Q$ is a symplectic manifold, it is in fact easy to show the following (see Proposition 4.1 of Meng, 2015), where we use $\tau_{T^{*} Q}: T T^{*} Q \rightarrow T^{*} Q$ to denote the natural tangent bundle projection.

Lemma 2.2.3. $S_{L}:=\left(T T^{*} Q, T Q, T \pi_{Q}, \theta_{Q}^{L}\right)$ is a special symplectic manifold, where $\alpha_{Q}: T T^{*} Q \rightarrow$ $T^{*} T Q$ is the relevant symplectomorphism required by Definition 2.2.1. $\theta_{Q}^{L}$ is the pullback of the canonical Liouville form $\theta_{T Q}$ on $T^{*} T Q$ along $\alpha_{Q}$, and the symplectic form on $T T^{*} Q$ is given by $\Omega_{Q}=d \theta_{Q}^{L}$.

Lemma 2.2.4. $S_{H}:=\left(T T^{*} Q, T^{*} Q, \tau_{T^{*} Q}, \theta_{Q}^{H}\right)$ is a special symplectic manifold, where $\beta_{Q}: T T^{*} Q \rightarrow$ $T^{*} T^{*} Q$ is the relevant symplectomorphism required by Definition 2.2.1. $\theta_{Q}^{H}$ is the pullback of the canonical Liouville form $\theta_{T^{*} Q}$ on $T^{*} T^{*} Q$ along $\beta_{Q}$, and the symplectic form on $T T^{*} Q$ is $\Omega_{Q}=d \theta_{Q}^{H}$.

It is furthermore easy to see that $\Omega_{Q}=d \theta_{Q}^{H}=d \theta_{Q}^{L}$.

$S_{L}$ is called the Lagrangian special symplectic manifold, and $S_{H}$ is called the Hamiltonian special symplectic manifold. As this terminology suggests, we now have the resources to discuss (a simple cas ${ }^{13}$ of how Tulczyjew lifts mechanics from $T Q$ and $T^{*} Q$, to $T^{*} T Q$ and $T^{*} T^{*} Q$ respectively.

A Hamiltonian T1-theory can be defined using the notion of a special symplectic manifold $S_{H}$. First, note that the Hamiltonian $H: T^{*} Q \rightarrow \mathbb{R}$ is a generating function for the Lagrangian submanifold $\mathcal{D}_{H}:=\operatorname{Graph}(d H)$ in $T^{*} T^{*} Q \sqrt{14}$ Furthermore, since symplectomorphisms preserve Lagrangian submanifolds, $\mathcal{E}_{H}:=\beta_{Q}^{-1}\left(\mathcal{D}_{H}\right)$ is also a Lagrangian submanifold in $T T^{*} Q$. Keeping in mind the structure of the special symplectic manifold $S_{H}$, we can think of both $\mathcal{D}_{H}$ and $\mathcal{E}_{H}$ as geometric representations of a Hamiltonian T1-theory, i.e. the analogs of a Hamiltonian $H$ in standard classical mechanics.

\footnotetext{
${ }^{13}$ In particular, we only consider the case where $H$ and $L$ are functions of an entire manifold. See [Meng, 2015] for the details of the case where $H$ and $L$ are restricted to proper submanifolds.

${ }^{14} \mathrm{In}$ our notation, $\operatorname{Graph}(f)$ is the set of points of the form $(x, f(x))$.
} 
This point of view is vindicated because $\beta_{Q}$ ensures that a curve in $\mathcal{E}_{H}$ satisfies Hamilton's equations when it is mapped to a curve in $\mathcal{D}_{H}{ }^{15}$ To see this, consider an arbitrary curve $c$ with coordinates $c=(q, p)$ on $T^{*} Q$, which gets lifted to a curve $c^{\prime}=\left(q, p, q^{\prime}, p^{\prime}\right)$ on $T T^{*} Q$ (where $(\cdot)^{\prime}$ should be interpreted as a time derivative). If $c^{\prime}$ is restricted to the Lagrangian submanifold $\mathcal{E}_{H}$, then $\beta$ maps it to a curve on $\mathcal{D}_{H}$ via:

$$
\left(q, p, q^{\prime}, p^{\prime}\right) \mapsto\left(q, p,-p^{\prime}, q^{\prime}\right) .
$$

But since points of $\mathcal{D}_{H}$ are of the form $(q, p, \partial H / \partial q, \partial H / \partial p)$, we see that such a curve must satisfy Hamilton's equations.

Similarly, a Lagrangian theory can be defined using the special symplectic manifold $S_{L}$. Again one notes that given a Lagrangian $L: T Q \rightarrow \mathbb{R}, \mathcal{D}_{L}:=\operatorname{Graph}(d L)$ is a Lagrangian submanifold of $T^{*} T Q$, and thus $\mathcal{E}_{L}:=\alpha_{Q}^{-1}\left(\mathcal{D}_{L}\right)$ is a Lagrangian submanifold of $T T^{*} Q$. We can then think of $\mathcal{D}_{L}$ and $\mathcal{E}_{L}$ as representations of a Lagrangian theory, because by a calculation similar to that of the Hamiltonian case, $\alpha_{Q}$ ensures that a curve on $\mathcal{D}_{L}$ satisfies the Euler-Lagrange equations (mutatis mutandis for a curve on $\varepsilon_{L}$ ).

In Tulczyjew's framework, a generalized Legendre transformation is said to exist when $\alpha_{Q}^{-1}\left(\mathcal{D}_{L}\right)=$ $\beta_{Q}^{-1}\left(\mathcal{D}_{H}\right)$. In other words, the criterion of T1-equivalence between $\mathcal{D}_{H}$ and $\mathcal{D}_{L}$ is that their respective images under $\beta_{Q}^{-1}$ and $\alpha_{Q}^{-1}$ are equal. Alternatively, we can view this equivalence as given by the symplectomorphism $\beta_{Q} \circ \alpha_{Q}^{-1}=\kappa_{T Q}$ which takes $\mathcal{D}_{L}$ to $\mathcal{D}_{H}$. It is easy to show that this map $\kappa_{T Q}$ projects down to the usual Legendre transformation (i.e. eq. (2)) in a sense made precise by Proposition 3.5 of [Tulczyjew, 1977].

We conclude this section by discussing how Tulczyjew's framework satisfies S1 (cf. Section 1.3), i.e. provides an appropriate notion of mathematical equivalence between Hamiltonian and Lagrangian mechanics. First, it exhibits Hamiltonian and Lagrangian theories (in the sense of T1) as the same type of mathematical object: $H$ and $L$ are lifted into Lagrangian submanifolds of $T^{*} T^{*} Q$ and $T^{*} T Q$ respectively, which are then mapped to Lagrangian submanifolds of $T T^{*} Q$. Second, these Lagrangian submanifolds in $T T^{*} Q$ are shown to coincide iff there is a (standard) Legendre transformation between $H$ and $L$.

We also emphasize that Tulczyjew's framework provides an attractive answer to the question of 'What structure gets preserved in a Legendre transformation?' This is best seen within the context of the extended symplectic category ExtSymp, whose objects are symplectic manifolds, and whose morphisms are canonical relations ${ }^{16}$ In ExtSymp, one can view the Lagrangian submanifolds of an object $O$ as its generalized 'points', i.e. as arrows from the terminal object (a point regarded as a symplectic manifold) to $O$. It follows that both $T^{*} T^{*} Q$ and $T^{*} T Q$ are objects of ExtSymp, whose appropriately generated 'points' are Hamiltonian and Lagrangian T1-theories respectively these objects can thus be seen as 'spaces of theories' ${ }^{17}$ Tulczyjew's framework tells us that certain isomorphisms in ExtSymp (viz. those symplectomorphisms defined by the Tulczyjew Triple) play the role of 'a mathematical standard of equivalence' between spaces of theories. Furthermore, such

\footnotetext{
${ }^{15}$ Conversely, one can start with a curve on $\mathcal{D}_{H}$ and show that it satisfies Hamilton's equations when it is mapped to $\mathcal{E}_{H}$.

${ }^{16}$ A canonical relation between two symplectic manifolds $M$ and $N$ is defined as a Lagrangian submanifold of the 'twisted product' $M \times \bar{N}$, where $\bar{N}$ means ' $N$ equipped with its opposite symplectic form'. For more details on the definition of ExtSymp and the technical difficulties involved in this construction, see Weinstein, 2009.

${ }^{17}$ This point of view can be elaborated by defining the co-slice category pt/ExtSymp, whose objects are canonical relations between a point and a symplectic manifold.
} 
isomorphisms preserve the structure of theories (i.e. Lagrangian submanifolds) in virtue of their geometrical structure.

\section{Equivalence regained}

In this section we explain how the Tulczyjew framework provides a good notion of theoretical equivalence between Lagrangian and Hamiltonian mechanics, i.e. how it fulfills desideratum S2, as discussed in Section 1.3. In particular we explain how $T T^{*} Q$ with its two special symplectic structures can be thought of as a common definitional extension of $T Q$ and $T^{*} Q$ in two distinct ways, one corresponding to an understanding of a theory in the sense of T1 and the other to T2.

\subsection{Common Definitional Extensions}

We briefly review the notion of a common definitional extension from (first-order) model theory which was perhaps first introduced in the philosophy of science by Glymour, 1970. Recall that a (first-order) theory $\overline{\mathbb{T}}$ (over a signature $\bar{\Sigma}$ ) is called a conservative extension of $\mathbb{T}$ (over a signature $\Sigma \subset \bar{\Sigma}$ ) if for any $\Sigma$-formula $\phi, \overline{\mathbb{T}}=\phi$ if and only if $\mathbb{T} \models \phi-$ in other words, if $\overline{\mathbb{T}}$ proves nothing over above what is already provable in the language of $\mathbb{T}$ (i.e. $\Sigma$ ) by $\mathbb{T}$ itself.

Definition 3.1.1. Let $\mathbb{T}$ be a first-order theory over a signature $\Sigma$ and let $\overline{\mathbb{T}}$ be a theory over a signature $\bar{\Sigma}$ possibly extending $\Sigma$. We say that $\overline{\mathbb{T}}$ is a definitional extension of $\mathbb{T}$ if the following two conditions hold:

1. $\overline{\mathbb{T}}$ is a conservative extension of $\mathbb{T}$

2. For any $\bar{\Sigma}$-formula $\psi$ there is a $\Sigma$-formula $\phi$ such that $\overline{\mathbb{T}} \models \phi \leftrightarrow \psi$.

It is easy to see that conditions (1) and (2) in Definition 3.1.1 are equivalent to requiring that every (non-logical) symbol in $\bar{\Sigma}$ that is not in $\Sigma$ is definable (in $\overline{\mathbb{T}}$ ) in terms of a $\Sigma$-formula 18 What this means is that $\overline{\mathbb{T}}$ differs from $\mathbb{T}$ only in giving explicit names to some notions are already implicitly defined in $\mathbb{T}$.

We will find it useful to denote the relation of $\mathbb{T}$ to $\overline{\mathbb{T}}$ by an arrow $i: \mathbb{T} \rightarrow \overline{\mathbb{T}}$ where $i$ is to be understood as the interpretation of $\mathbb{T}$ into $\overline{\mathbb{T}} 19$ With this in mind, we can then define the following notion of equivalence for first-order theories:

Definition 3.1.2. We say that $\mathbb{T}_{1}$ and $\mathbb{T}_{2}$ are definitionally equivalent if they have a common definitional extension $\mathbb{T}_{12}$, i.e. if there is a theory $\mathbb{T}_{12}$ that is a definitional extension of both $\mathbb{T}_{1}$ and

\footnotetext{
${ }^{18}$ This also assumes that we are talking about single-sorted theories. It is possible, but not entirely straightforward, to extend these notions to multi-sorted theories. Indeed, the (signature-independent) notion of Morita equivalence established in Johnstone, 2007 is just such an extension and forms the basis of O. Caramello's "Unification Project" (see Caramello, 2010 for an overview and Caramello, 2012 for issues related to definability). Closer to our concerns, see Tsementzis, 2015 for an explanation of how this topos-theoretic notion of Morita equivalence corresponds to a generalization of the notion of common definitional extension considered here first defined in Barrett and Halvorson, 2015b. Also see Barrett and Halvorson, 2015a for evidence about how the latter notion can prove useful in the philosophy of science. We will, however, say no more about this here, and maintain a working assumption that all the theories we are considering are single-sorted.

${ }^{19}$ Strictly speaking, we can think of $i$ as the inclusion of the signature of $\Sigma$ of $\mathbb{T}$ into the signature $\bar{\Sigma}$ of $\overline{\mathbb{T}}$ and the interpretation of $\mathbb{T}$ into $\overline{\mathbb{T}}$ that it induces.
} 
$\mathbb{T}_{2}$. This property can be expressed in the following 'span' diagram:

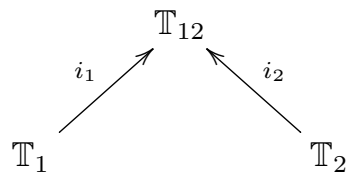

If $\mathbb{T}_{1}$ and $\mathbb{T}_{2}$ have a 'common definitional extension' then every notion definable in $\mathbb{T}_{1}$ is also definable in $\mathbb{T}_{2}$, albeit in a possibly different vernacular. For instance, the imperial and the metric systems are in sensu stricto different theories of distance even though, intuitively, they are theories about the 'same' notion of distance. This 'sameness' can be formalized as 'definitional equivalence', meaning that we can systematically translate one theory into the other ${ }^{20}$ In short, definitional equivalence captures the situation when two theories $\mathbb{T}_{1}$ and $\mathbb{T}_{2}$ differ only syntactically, but express the same propositions. As such, we take Definition 3.1 .2 to provide a good notion of theoretical equivalence.

There is a slight subtlety here that we ought to remark on. The interpretations $i_{1}$ and $i_{2}$ above are not themselves functions, but they do induce functions $i_{1}^{*}\left(\right.$ resp. $i_{2}^{*}$ ) between the class of models of $\mathbb{T}_{1}$ (resp. $\mathbb{T}_{2}$ ) and the class of models of $\mathbb{T}_{12}{ }^{21}$ It is then reasonable to ask whether having a common definitional extension means that the images of these two induced maps $i_{1}^{*}$ and $i_{2}^{*}$ coincide - all the more so because in our analogy below the images of the relevant Hamiltonians and Lagrangians under the Tulczyjew construction do, in fact, coincide (as Lagrangian submanifolds).

The answer is not entirely straightforward. On the one hand, it is true that the images of $i_{1}^{*}$ and $i_{2}^{*}$ do coincide in the classical definition in the following sense: given a model $\mathcal{M}_{1}$ of $\mathbb{T}_{1}$ we get a model $i_{1}^{*}\left(\mathcal{M}_{1}\right)$ of $\mathbb{T}_{12}$ which coincides with a model $i_{2}^{*}\left(\mathcal{M}_{2}\right)$ for some model $\mathcal{M}_{2}$ of $\mathbb{T}_{2}$ (indeed this is how the correspondence between models of $\mathbb{T}_{1}$ and models of $\mathbb{T}_{2}$ is defined, given a common definitional extension). On the other hand, in the extended notion of common definitional extension which is the proper "logical version" of Morita equivalence (see Barrett and Halvorson, 2015b, Tsementzis, 2015]) we no longer have strict coincidence: the images coincide only in an appropriate up-to-isomorphism sense. We will rest content that our analogy is compatible with both notions even though only as a special case as far as the latter notion is concerned.

Now let $\operatorname{Mod}(\mathbb{T})$ stand for the class of models of a first-order theory $\mathbb{T}$ and write $U(\mathcal{M})$ for the underlying set (or 'universe') of a given $\mathcal{M} \in \operatorname{Mod}(\mathbb{T})$. It is easy to show the following (cf. e.g. Hodges, 1993 p.61-62.)

Proposition 3.1.3 (Andreka et al., 2005). $\mathbb{T}_{1}$ and $\mathbb{T}_{2}$ are definitionally equivalent if and only if there is a bijection $m: \operatorname{Mod}\left(\mathbb{T}_{1}\right) \rightarrow \operatorname{Mod}\left(\mathbb{T}_{2}\right)$ such that for all $\mathcal{M} \in \operatorname{Mod}\left(\mathbb{T}_{1}\right)$ we have $U(m(\mathcal{M}))=$ $U(\mathcal{N})$ and all $\mathcal{N}$-predicates are uniformly definabl ${ }^{22}$ in terms of only $m(\mathcal{N})$-predicates and vice

\footnotetext{
${ }^{20}$ One must however be careful not to confuse 'mutual definability' with 'definitional equivalence'. The former property is strictly weaker than the latter. Definitionally equivalent theories are always mutually definable but there are theories that are mutually definable but not definitionally equivalent, cf. Andreka et al., 2005. A better way to put the above example would be to say that the theory of distances in miles and the theory of distances in nautical miles can both be translated as a theory of distance in meters (which provides their common definitional extension.)

${ }^{21}$ These are the "expansion" maps. There are also obvious "reduct" maps in the other direction that send a model $\mathcal{M}$ of $\mathbb{T}_{12}$ to a model $\mathcal{M} \mid L_{1}$ (where $L_{1}$ is the language of $\mathbb{T}_{1}$ ) by simply forgetting extra structure in $\mathcal{M}$ which is there as a result of interpreting $L_{1} \cup L_{2}$.

${ }^{22}$ A predicate $P \in \Sigma_{1}$ for a theory $\mathbb{T}_{1}$ is uniformly definable in terms of predicates $Q_{1}, \ldots, Q_{n} \in \Sigma_{2}$ for some theory $\mathbb{T}_{2}$ if there exists a unique $\left(n+1\right.$-place open) formula $\phi$ (in the ambient set theory) such that for any model $\mathcal{M}$ of $\mathbb{T}_{1}$, $P^{\mathcal{M}}=\left\{x \mid \phi\left(x, Q_{1}^{\mathcal{M}}, \ldots, Q_{n}^{\mathcal{M}}\right\}\right.$, assuming, of course that $\mathcal{M}$ is a model also of $\mathbb{T}_{2}$ possibly under some correspondence, e.g. $m$ in the statement of the Proposition.
} 
versa.

Now suppose we rephrase the right hand side of the biconditional in Proposition 3.1 .3 as

$$
\operatorname{Mod}\left(\mathbb{T}_{1}\right) \simeq \operatorname{Mod}\left(\mathbb{T}_{2}\right)
$$

and read ' $\simeq$ ' as 'is equivalent to' - then Proposition 3.1 .3 says that two theories are definitionally equivalent iff their classes of models are equivalent. Even though the precise meaning of 'equivalence' has only been specified by Proposition 3.1.3 itself, one can already see that there is a formal similarity between this notion and that of a 'Morita equivalence' of rings ${ }^{23}$ Indeed, we can take definitional equivalence to be a kind of Morita equivalence for theories.

We thus see that there are really two aspects of definitional equivalence. One takes place on the syntactic side, as expressed in Definition 3.1.2. The other concerns the semantic side, and expresses the fact that two theories are equivalent if their 'classes' of models are 'equivalent' ${ }^{24}$ As such, the notion of a common definitional extension in some sense fits with both the semantic and the syntactic view of theories, construing each in such a way as to make them appear merely as opposite sides of the same coin. This, as far as we are concerned, is one of the main conceptual advantages of this approach to the question of theoretical equivalence.

But let us go back to (CM-Equiv): we want to use the above-sketched notions to demonstrate the equivalence of Hamiltonian and Lagrangian mechanics. In particular, we want to show that Hamiltonian and Lagrangian mechanics have a common definitional extension, which in turn induces an 'equivalence' between their respective 'classes' of models. We will do so by arguing that the Tulczyjew triple for an arbitrary configuration space $Q$ can be used to exhibit $T T^{*} Q$ as a common definitional extension of Lagrangian and Hamiltonian mechanics. 25

The basic idea is that there are two span-like ${ }^{26}$ constructions implicit in Tulczyjew's framework, which are analogous to the span diagram in Definition 3.1.2. Natural operations on projection maps yield the span

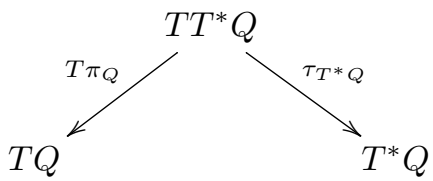

\footnotetext{
${ }^{23}$ Two commutative rings are Morita equivalent if their categories of (left) modules are equivalent. The analogy alluded to becomes clear if one takes modules of a ring to resemble models of some (fixed) theory. Or, alternatively, if one takes models of a theory to resemble representations of some structure.

${ }^{24}$ The ambiguous term 'classes' is used here in order to remain non-committal (and suggestive) about what kind of structure we take these classes $\operatorname{Mod}(\mathbb{T})$ to carry. Similarly, we put the term 'equivalence' in scare quotes so as to remain non-committal about exactly which notion of equivalence we have in mind here.

${ }^{25}$ Ideally, of course, we would do this by exhibiting each component as a first-order theory and then formally proving that they are definitionally equivalent. Indeed, it is prima facie unclear whether such a first-order axiomatization is even possible: continuity - being a second-order notion - will necessarily have to be dealt with either by an axiom schema or methods going beyond first-order logic. That said, there have been many attempts to bypass this difficulty by using a more 'synthetic' approach and indeed this has resulted in explicit axiomatizations of classical mechanics. Among others, Lawvere and Truesdell offer examples of such an approach, but we will not investigate this direction any further. Suffice it to say that such an approach to mechanics - although conceptually attractive - is too far removed from the standard framework in which Tulczyjew's ideas are developed. We proceed, rather, by analogy, leaving such a technical undertaking for the future.

${ }^{26} \mathrm{~A}$ span in a category $\mathcal{C}$ from objects $A$ to $B$ consists of a pair of maps $A \leftarrow C \rightarrow B$. A paradigmatic span would be that given by the product (in a category with products) $A \times B$ together with the two projections. A cospan is exactly the same thing except with the arrows reversed (i.e. going 'into' $C$.) The diagram associated to Definition 3.1 .2 is an example of a cospan - if one ignores the fact that the category in which it leaves has not been specified.
} 
which in turn extends to the following double-span by means of Tulzyjew's Triple and additional projections:

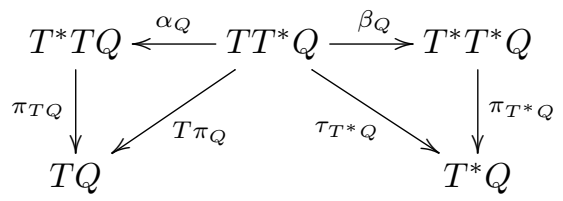

In the next section (Section 3.2), we shall see that these spans can be interpreted as relating Lagrangian and Hamiltonian mechanics to a common definitional extension, thus providing a translation between them.

\subsection{A First Analogy}

Our first analogy (summarized in Table 1) is between the span-like notion of equivalence expressed in Definition 3.1.2 (i.e. the existence of a common definitional extension), and T1-equivalence as expressed by the Tulczyjew Triple.

\begin{tabular}{l|r}
$\mathbb{T}_{1}$ & A hyperregular Hamiltonian $H: T^{*} Q \rightarrow \mathbb{R}$ \\
$\mathbb{T}_{2}$ & A hyperregular Lagrangian $L: T Q \rightarrow \mathbb{R}$ \\
$\Sigma_{1}$ & $T^{*} Q$ (cotangent bundle of a configuration space $Q$ ) \\
$\Sigma_{2}$ & $T Q$ (tangent bundle of a configuration space $Q$ ) \\
$\mathbb{T}_{12}$ & $\alpha_{Q}^{-1} \circ d L: T Q \rightarrow T T^{*} Q$ \\
$i_{1}: \mathbb{T}_{1} \rightarrow \mathbb{T}_{12}$ & The Lagrangian submanifold $\mathcal{E}$ of $T T^{*} Q$ generated by $H$ and $L$ \\
$i_{2}: \mathbb{T}_{2} \rightarrow \mathbb{T}_{12}$ & $\beta_{Q}^{-1} \circ d H: T^{*} Q \rightarrow T T^{*} Q$
\end{tabular}

Table 1: Analogy between 'Common Definitional Extension' and T1-equivalence

In this analogy, we take a T1-theory $\mathbb{T}_{1}$ of Hamiltonian mechanics to be given by a Hamiltonian $H: T^{*} Q \rightarrow \mathbb{R}$, and a 'signature' $\Sigma_{1}$ for this theory to be given by the cotangent bundle $T^{*} Q$ of our given configuration space $Q$. (Note that in order to expand T1 into a theory in the sense of 'equations of motion', we should include vector fields on $T^{*} Q$ as part of the syntax; cf. footnote 9.) Similarly, a T1-theory $\mathbb{T}_{2}$ of Lagrangian mechanics is given by a Lagrangian $L: T Q \rightarrow \mathbb{R}$, and the signature $\Sigma_{2}$ of $\mathbb{T}_{2}$ is given by the tangent bundle $T Q$.

By invoking Tulczyjew's framework and Lemmas 2.2.4 and 2.2.3 we know that both $H$ and $L$ uniquely generate (i.e. are generating functions of) the Lagrangian submanifolds $\mathcal{D}_{H} \subset T^{*} T^{*} Q$ and $\mathcal{D}_{L} \subset T^{*} T Q$, which can in turn be pulled back by $\beta_{Q}^{-1}$ and $\alpha_{Q}^{-1}$ respectively, to give the Lagrangian submanifolds $\mathcal{E}_{H}$ and $\mathcal{E}_{L}$ in $T T^{*} Q$. We are thus in a position to see how to formulate an analog of 'common definitional extension' for T1-equivalence: since $\mathbb{T}_{1} \equiv H$ and $\mathbb{T}_{2} \equiv L$ are equivalent precisely when $\mathcal{E}_{H}=\mathcal{E}_{L}=\mathcal{E}$, we shall take this common Lagrangian submanifold $\mathcal{E}$ to be the analog of a common definitional extension $\mathbb{T}_{12}$ for $\mathbb{T}_{1}$ and $\mathbb{T}_{2}$, whose signature $\Sigma_{12}$ is taken to be $T T^{*} Q$. We can think of the maps $\alpha_{Q}^{-1} \circ d L: T Q \rightarrow T T^{*} Q$ and $\beta_{Q}^{-1} \circ d H: T^{*} Q \rightarrow T T^{*} Q$ as inclusions of $\Sigma_{1}$ and $\Sigma_{2}$ into $\Sigma_{12}$, which in turn induce the 'interpretations' $i_{1}: \mathbb{T}_{1} \rightarrow \mathbb{T}_{12}$ and $i_{2}: \mathbb{T}_{2} \rightarrow \mathbb{T}_{12}$.

From this analogy, we see that the symplectomorphism $\kappa_{T Q}: T^{*} T Q \rightarrow T^{*} T^{*} Q$ provides a 'definitional equivalence' between (a T1-equivalent pair) $H$ and $L$. Let us now consider the implications of this equivalence for the inter-translatability of dynamical curves on $T^{*} Q$ (which satisfy Hamilton's equations) to dynamical curves on $T Q$ (which satisfy the Euler-Lagrange equations), and vice versa. 
A dynamical curve on $T^{*} Q$ lifts to a curve on a Lagrangian submanifold $\mathcal{D}_{H} \subset T^{*} T Q$, whose pullback via $\beta_{Q}^{-1}$ to a curve $\gamma$ on $\mathcal{E} \subset T T^{*} Q$ can be seen to satisfy Hamilton's equations. Furthermore, this same curve $\gamma$ can be seen to satisfy the Euler-Lagrange equations because it is the pullback via $\alpha_{Q}^{-1}$ of the lift of a dynamical curve on $T Q$. Jointly, these two observations allow us to translate back and forth between two descriptions of the same dynamical curve $\gamma{ }^{27}$ We thus see that the 'common definitional extension' provided by Tulczyjew's Triple ensures the definitional equivalence of dynamical curves on $T^{*} Q$ and $T Q$ respectively.

We end this section with a brief remark on the role that the canonicity of $\alpha$ and $\beta$ plays in the above translation manual. The canonicity of these maps can be interpreted as telling us that $\mathcal{D}_{L}, \mathcal{D}_{H}$, and $\mathcal{E}$ are all definable from each other without adding any further information. So for instance, even though $\mathcal{D}_{H} \subset T^{*} T^{*} Q$ does not allow us to explicitly describe vector fields on $T^{*} Q$, this information is in fact encoded as information about 1 -form fields on $T^{*} Q$, as shown by the canonical symplectomorphism $\beta{ }^{28}$

\section{$3.3 \quad$ A Second Analogy}

Our second analogy is between the notion of equivalence expressed after Proposition 3.1.3 and the use of Tulczyjew's framework to establish a T2-equivalence. Let us emphasize that (unlike Section 3.2 here we are taking theories $\mathbb{T}_{1}$ and $\mathbb{T}_{2}$ to be classes of models, where a model is a particular Lagrangian or Hamiltonian (given a fixed configuration space $Q$ ). The analogy can be summarized in the following table:

\begin{tabular}{l|r}
$\mathbb{T}_{1}$ & Hamiltonian Mechanics on $\mathrm{Q}$ \\
$\mathbb{T}_{2}$ & Lagrangian Mechanics on $\mathrm{Q}$ \\
$\operatorname{Mod}\left(\mathbb{T}_{1}\right)$ & Set of Hamiltonians $H: T^{*} Q \rightarrow \mathbb{R}$ \\
$\operatorname{Mod}\left(\mathbb{T}_{2}\right)$ & Set of Lagrangians $L: T Q \rightarrow \mathbb{R}$ \\
Equivalence between $\operatorname{Mod}\left(\mathbb{T}_{1}\right)$ and $\operatorname{Mod}\left(\mathbb{T}_{2}\right)$ & Correspondence induced by the Tulczyjew Triple
\end{tabular}

Table 2: Analogy between 'Equivalent classes of models' and T2-equivalence

\footnotetext{
${ }^{27}$ They also show the sense in which $\mathbb{T}_{12}$ can be regarded as a 'conservative extension' of $\mathbb{T}_{1}$ and $\mathbb{T}_{2}$ : a proposition (satisfaction of the dynamical equations) is true of a curve in $T^{*} Q$ or $T Q$ iff it is true of the corresponding curve $\gamma$ in $\mathcal{E} \subset T T^{*} Q$.

${ }^{28}$ One might say that the situation is analogous to the following. Suppose $\mathbb{T}_{g}$ is the theory of groups axiomatized over the signature $\Sigma_{g}=\{*, e\}$, i.e. using a 2-ary operation symbol and a constant symbol $e$ for the identity. Over $\mathbb{T}_{g}$ we cannot speak of an element $x$ of a model $G$ of $\mathbb{T}_{g}$ having an 'inverse' - we can certainly speak of there being a $y \in G$ such that $x * y=e$, but we do not yet have a 'word' for such a $y$. To rectify this, we may add a 1-place operation $(-)^{-1}$ to $\Sigma_{g}$ together with an explicit definition

$$
\forall x \forall y\left(y=x^{-1} \leftrightarrow y x=e\right)
$$

Call this new theory (over the expanded signature $\left.\Sigma_{g+}\right) \mathbb{T}_{g+}$. In a model $H$ of $\mathbb{T}_{g+}$ we may now freely speak of an element $h \in H$ having an 'inverse' since we now have added a 'word' for it, viz. the symbol $(-)^{-1}$. (This of course is also exactly the sense in which a definitional extension of a theory gives us new explicit terms to decsribe phenomena without adding any more content.) Similarly, in the case of classical mechanics, we are unable to ask whether a curve in a Lagrangian submanifold of $T^{*} T^{*} Q$ (for example) satisfies the "equations of motion" since there is no word for such a concept in $T^{*} T^{*} Q$. In particular, we need to talk about vector fields and this requires the passage to $T T^{*} Q$ via $\beta$. Crucially however, all the information that we need in order to define what it means to satisfy the "equations of motion" is already contained in $T^{*} T^{*} Q$, the same way that all the information that we need in order to define what it means for an element of $G$ to have an inverse is already contained in $G$ (insofar as it is understood as a model of $\mathbb{T}_{g}$.)
} 
In this analogy, we mean to consider all possible Lagrangians (respectively Hamiltonians) on the tangent (respectively cotangent) bundle of a configuration space simultaneously, thus establishing a correspondence between these two sets. Recall that in Tulczyjew's framework, a Lagrangian $L \in \operatorname{Mod}\left(\mathbb{T}_{2}\right)$ is the generating function for a Lagrangian submanifold $\mathcal{D}_{L} \subset T^{*} T^{*} Q$; similarly, a Hamiltonian $H \in \operatorname{Mod}\left(\mathbb{T}_{1}\right)$ is a generating function for a Lagrangian submanifold $\mathcal{D}_{H} \subset T^{*} T Q$. Thus, there is an analogy between talking about equivalent classes of models, and 'equivalent' classes of Lagrangian submanifolds $\left\{\mathcal{D}_{H}\right\}$ and $\left\{\mathcal{D}_{L}\right\}$. The latter 'equivalence' is given by the symplectomorphism $\kappa$, which preserves Lagrangian submanifolds and induces a bijection between $\{H\}$ and $\{L\}$.

One can attempt to view the second analogy as a trivial 'categorification' of the first analogy, in the sense that the category of Lagrangian submanifolds ( of $T^{*} T Q$ ) generated by Lagrangians on $T Q$ is equivalent (indeed isomorphic) to the category of Lagrangian submanifolds (of $T^{*} T^{*} Q$ ) generated by Hamiltonians on $T^{*} Q$. Note, however, that although this correspondence can be presented as having the superficial form of a 'categorical equivalence', it is essentially a bijection between two sets: the set of Lagrangian submanifolds representing Lagrangian mechanics on the one hand and the set of Lagrangian submanifolds representing Hamiltonian mechanics on the other. It is thus an interesting further question whether one can make this correspondence truly 'functorial' by defining some sort of interesting categorical structure on $\operatorname{Mod}\left(\mathbb{T}_{1}\right)$ and $\operatorname{Mod}\left(\mathbb{T}_{2}\right)$. In particular, can one find a pertinent (to classical mechanics) and mathematically interesting notion of a morphism between the Lagrangian submanifolds of a given symplectic manifold? When considered in full generality, this question is reminiscent of attempts to construct the Fukaya category ${ }^{29}$ of a symplectic manifold (which forms part of the mathematical basis for a more sophisticated duality, viz. mirror symmetry). However, it is not clear to us that such sophisticated constructions find applications in CM-Equiv.

With this in mind, let us return to the question of what Tulczyjew's framework adds to the usual Legendre transformation. After all, one can imagine a skeptic saying: 'The usual Legendre transformation establishes a bijection between Lagrangians and Hamiltonians on a fixed configuration space. And what you have described seems to accomplish nothing more than this, since there appears to be no extra categorical structure that makes this classical correspondence functorial in an interesting way.' If the skeptic were right, then our rearticulation of this correspondence in terms of symplectic geometry would merely be a superficial rephrasing, rather than a substantive change of perspective.

There are two things to say in response. First, the above correspondence is induced by maps $\left(\alpha_{Q}\right.$ and $\left.\beta_{Q}\right)$ that manifestly preserve all the relevant structure, viz. the symplectic structure of the manifolds under consideration. As explained in Section 2.2, all the mechanical information of interest has been re-expressed in terms of symplectic geometry; as such, preserving the symplectic structure preserves all the mechanically relevant structure. Furthermore (and as discussed at the end of Section 2.1), this is a conceptual improvement over the naive bijection induced by the usual Legendre transformation, since the latter does not provide any insight into how the 'mechanically relevant' structure is preserved.

Second, Tulczyjew's framework exhibits this correspondence in a natural manner, i.e. as independent of a particular configuration space, thus exhibiting a kind of canonicity that is lacking in the classical correspondence via the Legendre transformation (as we explained at the end of Section 2.1). More precisely, the Tulczyjew triple can be understood as a span of natural transformations between functors - we proceed to explain this point in the following Section.

\footnotetext{
${ }^{29}$ See e.g Auroux, 2013 for an introduction.
} 


\subsection{Naturality of the Tulczyjew Triple}

Recall that the Tulczyjew triple consists of the following (double) span diagram, defined for any differentiable manifold $Q$.

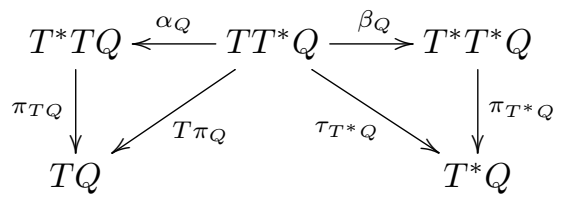

To anyone familiar with the notation of category theory, the top part of the diagram immediately raises the question: Are $\alpha$ and $\beta$ natural transformations between (suitably defined) functors? We proceed to explain how this question can be answered in the affirmative.

First, we must clarify which functors are acted upon by the putative natural transformations $\alpha$ and $\beta$. On first inspection there seems to be a variance issue: since the cotangent bundle functor $T^{*}: \mathbf{M a n}^{\mathrm{op}} \rightarrow$ Man is a contravariant functor and the tangent bundle functor $T:$ Man $\rightarrow$ Man is a covariant functor, we cannot meaningfully speak of a natural transformation $\beta: T T^{*} \Rightarrow T^{*} T^{*}$ since the domain is contravariant (being the composition of a contravariant followed by a covariant endofunctor) whereas the codomain is covariant (being the composition of two contravariant endofunctors. ${ }^{30}$

The solution is two-fold. Firstly, we will regard $T^{*} T^{*}$ and $T^{*} T$ as single functors rather than composites of previously defined functors. Secondly, we will 'cut down' on the domain category of these functors by considering only its associated groupoid, or "core" 31 Doing so gives us the following three functors, where $\mathbf{M a n}_{g}$ is the category of smooth manifolds and diffeomorphisms between them (i.e. the associated groupoid, or "core", of the category of smooth manifolds and smooth maps between them) and Symp is the category of symplectic manifolds and symplectomorphisms between them:

- $T^{*} T^{*}: \mathbf{M a n}_{g} \rightarrow$ Symp: $T^{*} T^{*} Q$ is given the canonical symplectic structure on $T^{*} Q$, where the latter is viewed as a smooth manifold.

- $T T^{*}: \mathbf{M a n}_{g} \rightarrow$ Symp: $T T^{*} Q$ is given the symplectic structure described e.g. by Meng, 2015 in Proposition 4.1, i.e. the canonical lift of the symplectic structure on $T^{*} Q$.

- $T^{*} T: \mathbf{M a n}_{g} \rightarrow$ Symp: $T^{*} T Q$ is again given by the canonical symplectic structure on $T Q$, where the latter is considered as a smooth manifold.

Strictly speaking we would also need to describe exactly what each of the three functors does on arrows. However, in the current set-up this is somewhat unnecessary: since all arrows in $\mathbf{M a n}_{g}$ are diffeomorphisms, and since all the symplectic structures considered in the definitions above are canonical, we know that diffeomorphic manifolds will give rise to canonically symplectomorphic constructions in each of the three cases above. As such, each diffeomorphism is sent by each of the functors to the canonical symplectomorphism it induces on each construction.

\footnotetext{
${ }^{30}$ We could of course speak of a dinatural transformation between such functors, but we will not pursue this line of thought here.

${ }^{31}$ In effect, what we are doing can also be understood as composing two functors, only because on arrows they will always give isomorphisms, in order to harmonize their variance we stipulate that sometimes the value of the composite functor is the quasi-inverse of the arrow that would have originally been selected. To make this thought technically precise, we need to consider these functors as 'inseparable'.
} 
Now we want to show that the maps that define the Tulczyjew triple are in fact natural transformations between the above-defined functors. To do so, it suffices to show that $\beta$ and $\kappa$ are natural transformations since $\alpha$ is defined as the composite $\kappa \circ \beta$. Now $\beta_{Q}$ is the canonical vector bundle isomorphism between the tangent bundle and the cotangent bundle of $T^{*} Q$. As with any smooth manifold, these maps are natural transformations. On the other hand, $\kappa$ is the double vector bundle isomorphism described in Section 2.2. That $\kappa$ is a natural transformation is more difficult to establish, because on the face of it, its definition seems to rely on the choice of a particular connection on the fiber bundle on which it is being applied. However the construction itself turns out to be independent of the particular choice of connection, as shown by the local formula for $\kappa$. It is then routine to check that the appropriate naturality diagram does indeed commute.

With the above in mind, the Tulczyjew triple can thus be understood as involving the following three functors:

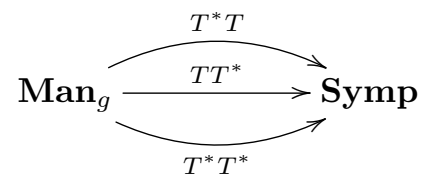

and the following two natural transformations:

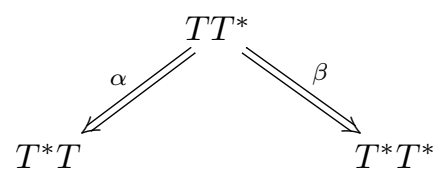

This span of natural transformations further reinforces the idea that there is something analogous to a common definitional extension going on here, i.e. that $T T^{*}$ (viewed as a 'theory') is analogous to a common definitional extension of $T^{*} T$ and $T^{*} T^{*}$ (also viewed as 'theories'). Although the arrows here look like they are going the 'wrong way', note first that they are to be thought of as the arrows induced by interpretations between theories (which go in the opposite direction) ${ }^{32}$ Furthermore, each of these arrows is in really an isomorphism (i.e. $\alpha$ and $\beta$ are natural isomorphisms) since each of their components is a symplectomorphism. As such, they are invertible and so this span can equally well be understood as a co-span.

At this point, it is instructive to consider what the above construction accomplishes over and above the more familiar approach of sticking with $T$ and $T^{*}$ (the tangent and cotangent bundle functors), fixing the discrepancy in their variance by restricting to $\mathbf{M a n}_{g}$ as above, and then saying that they are (non-canonically) isomorphic as vector bundles. If one takes the latter approach, one has to live with a major asymmetry between $T$ and $T^{*}$, viz. that $T^{*}$ naturally lands in Symp (since the Liouville form can equip any cotangent bundle with the structure of a symplectic manifold) whereas $T$ does not. By constrast, Tulczyjew's approach turns this asymmetry into a symmetry at a higher level, i.e. by hitting everything with a cotangent functor and thus absorbing all of mechanics into symplectic geometry.

Next, what does naturality mean in this context, and what does it tell us about inter-theoretic equivalence? To answer these questions, it would be helpful to say something about the connection between naturality in the category-theoretic sense and 'uniform definability' in the more traditional

\footnotetext{
${ }^{32}$ This is from the point of view that takes theories to be categories and models to be functors. Given an interpretation functor $i: \mathcal{C} \rightarrow \mathcal{D}$ between two theories (categories), for any given Set-model $M: \mathcal{D} \rightarrow$ Set we get a model $i^{*} M$ of $\mathcal{C}$ induced by precomposition with $i$.
} 
set-theoretic sense. Very roughly, naturality in the category-theoretic sense is a kind of uniform definability in the set-theoretic sense. This idea (and its limitations) has been made precise in Hodges and Shelah, 1986, whose work suggests that the naturality of the Tulczyjew triple can be cashed out in more traditional model-theoretic terms as a 'uniform definability' result. From this perspective, Tulczyjew's set-up can be understood as explaining the sense in which both Lagrangians on a tangent bundle and Hamiltonians on a cotangent bundle are definable (and equivalent) irrespective of the configuration space on which each bundle is defined. In other words, 'uniformity' amounts to the following property: given an arbitrary configuration space $Q$ (i.e. a differentiable manifold) we can describe how Hamiltonians on its cotangent bundle will correspond to Lagrangians on its tangent bundle without mentioning any specific properties of $Q$. This restores an element of canonicity - cf. our discussion of S2 above - that is lacking in the correspondence via the usual Legendre transformation.

\section{Further connections and concluding morals}

Having explained how Tulczyjew's framework yields a 'theoretical equivalence' between (hyperregular) Hamiltonian and Lagrangian mechanics, we now point out some tantalizing connections and analogies between this framework and more sophisticated dualities ${ }^{33}$

- Duality in classical field theory:

First, let us remind the reader that although many sophisticated dualities are theoretical equivalences between quantum theories, some of these dualities display a classical remnant at the level of the classical action. For instance, through the parent action approach, one can sometimes show that two different classical Lagrangians (with different sets of fields) are related by a Legendre transformation ${ }^{34}$ It may thus turn out to be the case that Tulczjew's framework provides a perspicuous language for articulating (the classical part of) these dualities.

- The 'geometrization' of theoretical equivalence:

It is a consequence of Tulczyjew's framework that the generalized Legendre transformation can be viewed as a Lagrangian submanifold of $T T^{*} Q \times \overline{T T^{*} Q}$; in other words the 'theoretical equivalence map' has been geometrized. On the other hand, recent work on $T$-duality shows that this far more sophisticated equivalence can be formalized along similar lines: the $T$ duality between generalized complex manifolds $M$ and $M^{\prime}$ can be thought of as a generalized submanifold of the product $M \times M^{\prime}{ }^{35}$ It is reassuring to see that two prima facie very different forms of theoretical equivalence share a common geometrical character.

- Possible analogs of objects representing a common definitional extension:

In Section 3.2, we saw that a Lagrangian submanifold in $T T^{*} Q$ plays the role of a common definitional extension for T1-theories in $T^{*} T Q$ and $T^{*} T^{*} Q$. An analogous scenario is not uncommon for sophisticated dualities: one often finds a mathematical structure $T_{12}$ that allows

\footnotetext{
${ }^{33}$ We should point out that Urs Schreiber's recent work (see section 2.10 of Schreiber, 2015]) may provide a general template for trying to forge such connections - its virtue is its great generality; on the other hand, the 'canonicity' of the Legendre transformation is made fully explicit in Tulczyjew's more concrete framework.

${ }^{34}$ See e.g. Hjelmeland and Lindstrom, 1997] and references therein for an introduction to the Parent Action approach.

${ }^{35}$ For a precise statement of this equivalence, see Section 6.2 of Cavalcanti and Gualtieri, 2011.
} 
one to translate between dual pairs $T_{1}$ and $T_{2}$. For instance, in the case of $S$-Duality, there is a 6 -dimensional $(2,0)$-theory that allows one to derive $S$-duality when compactified in 2 dimensions ${ }^{36}$ There is also an interesting disanalogy here: unlike the common definitional extension of Tulczyjew's framework, the 6D $(2,0)$-theory seems to have its own physical interpretation (as a quantum field theory).

These connections merit exploration, but space constraints force us to leave this task to the future. We now conclude by summing up the morals of our paper.

First, we take it that part of attaining a deep conceptual understanding of a duality is being able to make manifest the relevant (possibly geometric) structures preserved by such a theoretical equivalence. One would ideally like to move beyond mere naive correspondences; furthermore, one would aspire to some form of canonicity in one's construction of an equivalence. Tulczyjew's framework shows us how to achieve both for classical mechanics.

Second, no philosophical conclusions should be drawn from the apparent lack of isomorphisms between theories that are intuitively dual. As we have seen, part of finding an isomorphism is to find the 'correct' theoretical context for articulating theories - it is this search that should be the starting point for philosophical reflection.

Third, we have shown that concepts and analogies from logic and category theory (and metamathematical tools more generally speaking) can be fruitfully employed by philosophers to improve our understanding of the immensely mathematically sophisticated ways in which 'theoretical equivalences' are believed to obtain in contemporary physics: finding an analog of 'common definitional extension' in Tulczyjew's reconceptualization of classical mechanics is a case in point. While we readily concede that the Tulczyjew framework might not be the only way to build this analogy, we hold that it is an especially attractive one, not least because its implementation is so tightly aligned with modern mathematical thinking.

\section{Acknowledgments}

We thank Hans Halvorson, Jeremy Butterfield, Thomas Barrett, Guowu Meng, and two anonymous referees for discussions about the paper. This paper was first presented at a 'Dualities' workshop organized by Elena Castellani and Dean Rickles at the University of Florence, and we are especially pleased to acknowledge their encouragement and support, as well as feedback from the workshop participants. We also thank seminar audiences at Princeton and Oxford.

\section{References}

[Andreka et al., 2005] Andreka, H., Madarasz, J. X., and Nemeti, I. (2005). Mutual definability does not imply definitional equivalence, a simple example. Mathematical Logic Quarterly, 51:591-597.

[Auroux, 2013] Auroux, D. (2013). A beginner's introduction to Fukaya categories. ArXiv e-prints.

[Barrett and Halvorson, 2015a] Barrett, T. and Halvorson, H. (2015a). Glymour and Quine on theoretical equivalence. forthcoming in Journal of Philosophical Logic. Available at: http://philsciarchive.pitt.edu/11341/.

\footnotetext{
${ }^{36}$ See [Witten, 2007] and the discussion on p. 15 of Polchinski, 2015].
} 
[Barrett and Halvorson, 2015b] Barrett, T. and Halvorson, H. (2015b). Morita equivalence. http://arxiv.org/abs/1506.04675.

[Caramello, 2010] Caramello, O. (2010). The unification of mathematics via topos theory. arXiv:1006.3930.

[Caramello, 2012] Caramello, O. (2012). Universal models and definability. Mathematical Proceedings of the Cambridge Philosophical Society, 152(2):279-302.

[Cavalcanti and Gualtieri, 2011] Cavalcanti, G. R. and Gualtieri, M. (2011). Generalized complex geometry and T-duality. ArXiv e-prints.

[Curiel, 2014] Curiel, E. (2014). Classical mechanics is lagrangian; it is not hamiltonian. British Journal for the Philosophy of Science, 106:57-88.

[Glymour, 1970] Glymour, C. (1970). Theoretical realism and theoretical equivalence. PSA: Proceedings of the Biennial Meeting of the Philosophy of Science Association, (1):275-288.

[Grabowska and Grabowski, 2014] Grabowska, K. and Grabowski, J. (2014). Tulczyjew triples: From statics to field theory. arXiv:1306.2744v2.

[Halvorson, 2012] Halvorson, H. (2012). What scientific theories could not be. Philosophy of Science, $79(2): 183-206$.

[Hjelmeland and Lindstrom, 1997] Hjelmeland, S. E. and Lindstrom, U. (1997). Duality for the nonspecialist.

[Hodges, 1993] Hodges, W. (1993). Model Theory. Cambridge University Press.

[Hodges and Shelah, 1986] Hodges, W. and Shelah, S. (1986). Naturality and definability i. J. London Mat. Society, 1:1-12. doi: 10.1112/jlms/s2-33.1.1.

[Johnstone, 2007] Johnstone, P. (2007). Sketches of an Elephant: A topos theory Compendium. Oxford University Press.

[Meng, 2015] Meng, G. (2015). Tulczyjew's approach for particles in gauge fields. Journal of Physics A, 48(145201).

[North, 2009] North, J. (2009). The 'structure' of physics: A case study. Journal of Philosophy, 106:57-88.

[Polchinski, 2015] Polchinski, J. (2015). Dualities. SHPMP.

[Schreiber, 2015] Schreiber, U. (2015). Classical field theory via cohesive homotopy types. http://ncatlab.org/schreiber/show/Classical+field+theory+via+Cohesive+homotopy+types.

[Suppe, 1989] Suppe, F. (1989). The Semantic Conception of Theories and Scientific Realism. Urbana: University of Illinois Press.

[Suppe, 1998] Suppe, F. (1998). Scientific theories: An assessment of developments, 1969-1998. Philosophy of Science, 67:S102-S115. Supplement, Proceedings of the 1998 Biennial Meetings of the Philosophy of Science Association. Part II: Symposia Papers. 
[Tsementzis, 2015] Tsementzis, D. (2015). A syntactic characterization of Morita equivalence. Available at: http://arxiv.org/abs/1507.02302.

[Tulczyjew, 1974] Tulczyjew, W. M. (1974). Hamiltonian systems, lagrangian systems, and the legendre transformation. Symposia Mathematica, (14):247-258.

[Tulczyjew, 1977] Tulczyjew, W. M. (1977). The legendre transformation. Annales de L' I.H.P., Section A, (1):101-114. arXiv:1405.0748v1.

[Tulczyjew, 1996] Tulczyjew, W. M. (1996). Homogeneous lagrangian systems. Gravitation, Electromagnetism and Geometric Structures, (1):91-136. arXiv:math-ph/0404075v1.

[van Frassen, 1980] van Frassen, B. (1980). The Scientific Image. Oxford University Press.

[Weinstein, 1981] Weinstein, A. (1981). Symplectic geometry. Bull. Amer. Math. Soc. (N.S.), 5(1):113.

[Weinstein, 2009] Weinstein, A. (2009). Symplectic categories. arXiv:0911.4133v1 [math.SG] 20 Nov 2009.

[Witten, 2007] Witten, E. (2007). Conformal Field Theory In Four And Six Dimensions. ArXiv e-prints. 